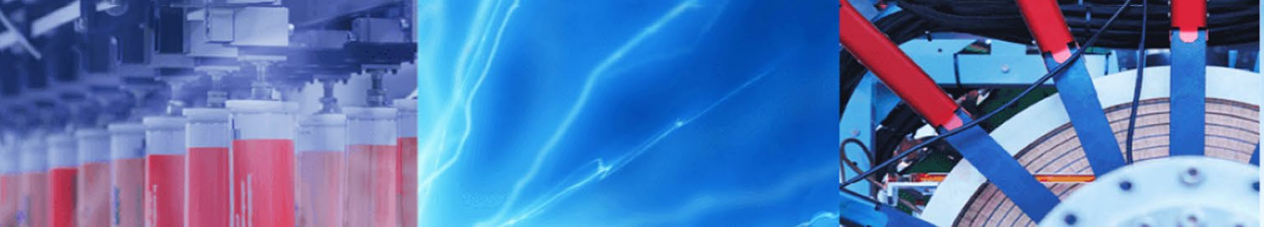

Research Article

\title{
Application of QuEChERS method for extraction of functional compounds
}

\author{
Mozhdeh Sarraf $^{1} \cdot$ Adel Beig-babaei $^{1} \cdot$ Sara Naji-Tabasi ${ }^{2}$
}

Received: 29 April 2020 / Accepted: 7 October 2020 / Published online: 22 October 2020

(c) Springer Nature Switzerland AG 2020

\begin{abstract}
Barberry has valuable compounds with high functional properties such as berberine. In this study, the QuEChERS method was applied for the extraction of berberine, phenolic, and antioxidant compounds of B.integerrima stem and compared with the maceration method. Also, the standard QuEChERS was modified to improve the extraction efficiency and application of this method in the food industries. The majority of the changes in the modified QuEChERS methods were the type of solvent, and removing salts and sorbents in the procedure. The average recovery of berberine was more efficient in maceration in comparison with the QuEChERS method. However, the ethanolic QuEChERS method had a positive effect on the extraction of phenolic compounds in comparison with the other applied methods. The modified QuEChERS method had a better yield to extract the phenolic compounds than the extraction of berberine compounds.
\end{abstract}

Keywords Barberry $\cdot$ QuEChERS · Maceration · Functional compounds

$\begin{array}{ll}\text { Abbreviations } \\ \text { dSPE } & \text { Dispersive solid-phase extraction } \\ \text { Gae } & \text { Gallic acid } \\ \mathrm{HPLC} & \text { High-performance liquid chromatography } \\ \mathrm{DPPH} & \text { 2,2-Diphenyl-1-picrylhydrazyl } \\ \mathrm{PSA} & \text { Primary and Secondary amine } \\ \mathrm{MgSO}_{4} & \text { Magnesium Sulfate } \\ \mathrm{NaCl} & \text { Sodium chloride }\end{array}$

\section{Introduction}

Although maceration is a standard method to extract functional compounds of plants, the development of novel approaches that are less commonly used in the food industry can help with the high capacity of extraction. QuEChERS is a Quick, Easy, Cheap, Effective, Rugged, and Safe method of extraction introduced for pesticide residues analysis in high moisture fruits and vegetables [1].
Recently, the technique is gained significant popularity in the analysis of a broad spectrum of analytes in a massive variety of samples [2-4]. QuEChERS, in addition to maintaining desirable results, increases sample yields, an issue of great importance. The advantages of QuEChERS include small amounts of material and solvent and decreasing the time spent to prepare the sample. This extraction method also has high efficiency [5].

Berberis belongs to the Berberidaceae family that includes 12 genera and over 666 species [6, 7]. This valuable plant contains essential compounds in different parts (flower, stem, fruit, shoot, leaf, and root), for instance, alkaloids, phenolic compounds, anthocyanin, flavonoids, and vitamins that have been used in traditional medicine the past to the present [8]. Bioactive phenols and alkaloids such as berberine have antioxidant activities that are in parts of barberry. Phenolic compounds have anti-pathogenic, anti-inflammatory, and antiseptic actions in the treatment of infectious, cancer, cardiovascular, and renal

$\triangle$ Adel Beig-babaei, a.beigbabaei@rifst.ac.ir; Sara Naji-Tabasi, s.najitabasi@rifst.ac.ir | ${ }^{1}$ Department of Food Chemistry, Research Institute of Food Science and Technology (RIFST), 91895-157.356, PO Box, Mashhad, Iran. ${ }^{2}$ Department of Food Nanotechnology, Research Institute of Food Science and Technology (RIFST), 91895-157.356, PO Box, Mashhad, Iran. 
diseases [9]. Also, Berberine has medicinal and therapeutic properties like antimicrobial, antioxidant, antihypertensive, antidiabetic, anticancer, anti-inflammatory, antidepressant, anti-diarrhea, cholagogues, hepatoprotective, hypolipidemic activities and Prevention of Alzheimer's and reduces total cholesterol, triglyceride levels [10-13].

In this research, it was used stems of B.integerrima var. asperma, which is a seedless type and commercially cultivated in Iran, to surveying amounts of phenol and berberine. Compounds like berberine can be found in different parts of barberry, especially roots and stems. However, using root as their resource causes irreparable losses in the plant [14].

According to the positive features of the QuEChERS method, the extraction of functional compounds of barberry with the QuEChERS were investigated in this present study. Some modifications were also done in the procedure to make this method usable in the food industry as an extraction method. Finally, this method was compared with maceration as a conventional method.

\section{Materials and methods}

\subsection{Chemicals, standard solutions and samples}

The stems of B.integerrima were collected from the botanical garden of Food Science and Technology Institute (Iran, Mashhad), in autumn. The plant parts were grinded (IKA, Germany), and dried under $40{ }^{\circ} \mathrm{C}$ in an oven (MemertUF55: Germany).

Acetonitrile (HPLC grade), phosphoric acid, and methanol of chromatographic grade were purchased from Merck Co. (Germany), and the standard berberine was supplied from Sigma-Aldrich Co. (Germany). Ethanol for maceration was prepared from Mojalali Co. (Iran). DPPH, folin-ciocalteu, $\mathrm{NaCl}, \mathrm{PSA}, \mathrm{C} 18$ tube, $\mathrm{MgSO}_{4}$ were supplied from Merck (Germany).

\subsection{Preparation of samples}

\subsubsection{The methodology based on maceration}

The maceration method was conducted based on Sarraf et al. (2020) [15]. According to this method, $15 \mathrm{~g}$ of barberry stems were milled by grinder (IKA: Germany) and mixed with $90 \%$ ethanol (the ratio of ethanol to powder was $11: 1)$. The extraction was done at $70^{\circ} \mathrm{C}$ for $3.36 \mathrm{~h}$ (141.6 $\mathrm{min}$ ) on a stirrer. Then extracts were filtered using Whatman paper (No. 41) and dried in an oven (MemertUF55: Germany) at $40^{\circ} \mathrm{C}(24 \mathrm{~h})$.

\subsubsection{The methodology based on QuEChERS procedure}

The QuEChERS technique consists of two steps; Extraction and Dispersive SPE (dSPE). The method relies on the combination of solvent and salts to separate the analyte from the sample into acetonitrile. $5 \mathrm{~g}$ of the sample was weighted in a tube, and shaken for $1 \mathrm{~min}$ with $10 \mathrm{~mL}$ of acetonitrile. Sorbents were added, and the tube was shaken in a vortex for $1 \mathrm{~min}$ and centrifuged for $5 \mathrm{~min}$ at $3000 \mathrm{~g}$. Then the supernatant was separated and cleaned by salts. The cleaned supernatant was shaken again, and centrifuged. $3 \mathrm{~mL}$ of the acetonitrile layer is removed from the extraction step and exposed to DSPE. The adsorbents compounds such as PSA remove unwanted interferences such as polar interferences, including sugars, organic acids, and pigments, and $\mathrm{C} 18$ is used to eliminate very non-polar interferences, such as fats, fatty acids $[2,4,16,17]$.

\subsubsection{The methodology based on modification of QuEChERS procure}

In this investigation, the standard QuEChERS method slightly was also modified to further development in the food industry. The changes were applied: (1) using ethanol solvent instead of acetonitrile, (2) the standard QuEChERS method without PSA sorbent, and (3) the standard QuEChERS method without salts.

According to the above cases, the majority of the modifications carried put on solvent, also salt, and sorbent formulations of the salting-out and the d-SPE steps [5].

Although using acetonitrile is preferred in the QuEChERS process due to making more environmentally friendly [17], it is a toxic solvent that using the solvent is banned for food intake. Therefore, ethanol was used instead of acetonitrile.

QuEChERS salt-free was conducted as a modified QuEChERS method to investigate the effect of salts on extraction of compounds. As the salts concentration and the Intrinsic nature influence on the stabilization or denaturation and salting-in or salting-out of some compounds such as proteins [18], the removal of salts was done as one of the methods. For instance, low salt concentration increases the solubility of proteins. But the high concentration of salt made salting out is generated that is due to the competition of salt and protein for absorption water.

In the other method, PSA was removed, because of the presence of the sorbet effects on organic acids and pigments. According to different researches, there are various types of pigments and organic acids in the barberry stems that have antioxidant properties. 


\section{Chemical analysis}

\subsection{Determination of berberine content}

The measurement of berberine content was conducted by Shimadzo LC- 2010 HPLC system (Kyoto, Japan) equipped with a UV-VIS detector $(346 \mathrm{~nm})$ and C-18 $(5.9 \times 1.81 \mathrm{inch})$ column. The Mobile phase contained $50 \%$ acetonitrile along with a $50 \%$ mix (1:1) of sodium perchlorate and (sodium) phosphate buffer. Injection volume was $40 \mu \mathrm{l}$ [19], and the mobile phase was used at a flow rate of $1.5 \mathrm{ml} \cdot \mathrm{min}^{-1}$ and column temperature $50^{\circ} \mathrm{C}$

The standard solutions of berberine were prepared at $1,5,10,15 \mathrm{ppm}$ concentrations. The standard curve was plotted by different standards. The $\mathrm{R}^{2}$ was obtained by 0.999 .

For the preparation of samples in the maceration method, $7 \mathrm{mg}$ of extract transferred to a $10 \mathrm{ml}$ volumetric flask and methanol (HPLC grade) was added (5 min ultrasound for complete dissolution) [20]. In the QuEChERS method, the samples injected directly with the solvent used (Fig. 1).

\subsection{Determination of DPPH radical scavenging activity}

$50 \mu \mathrm{l}$ of the extract was added to $5 \mathrm{~mL}$ of methanolic 2 , 2-diphenyl-1-picryl-hydrazyl-hydrate (DPPH) 0.004\%. Then it was kept in the dark place for $30 \mathrm{~min}$, and the absorbance of the solution was measured at $517 \mathrm{~nm}$ by spectrophotometer (Hach, USA) [21].

Inhibition $\%=\frac{100\left(A b s_{\text {blank }}-A b s_{\text {treatment }}\right)}{A b s_{\text {blank }}}$

\subsection{Determination of total phenolic content}

The amount of total polyphenols was assayed colorimetrically by the Folin-Ciocalteu method. $2.5 \mathrm{~mL}$ of
Fig. 1 The results of HPLC chromatogram of standard berberine (a) with standard QuEChERS (b)

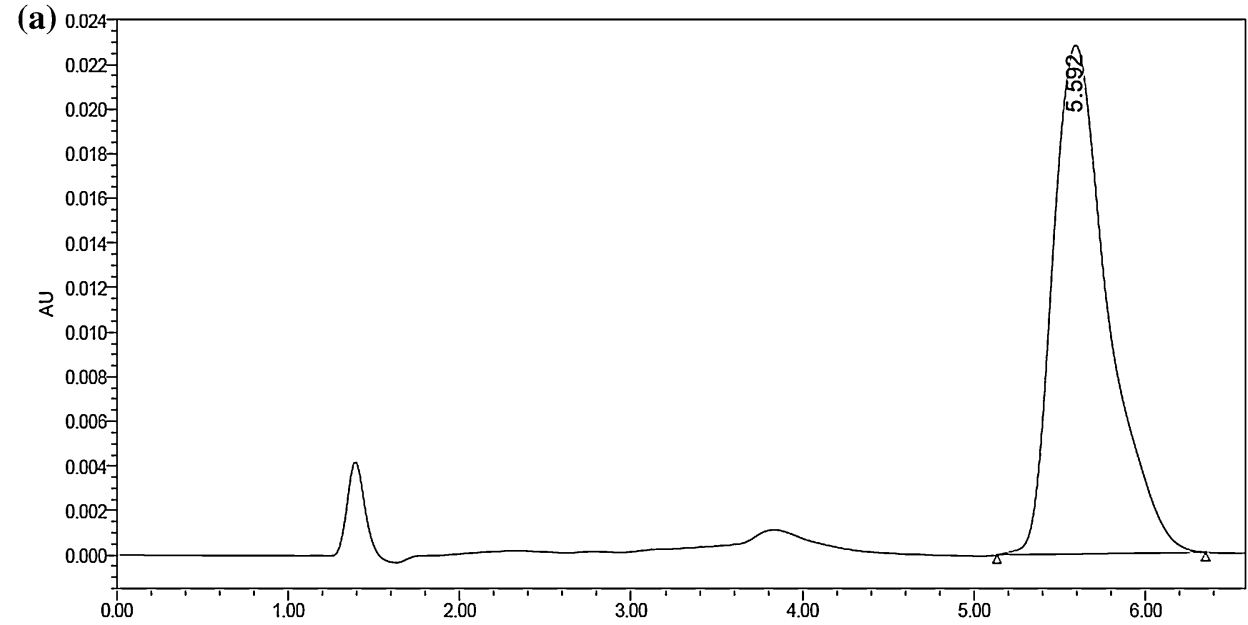

(b)

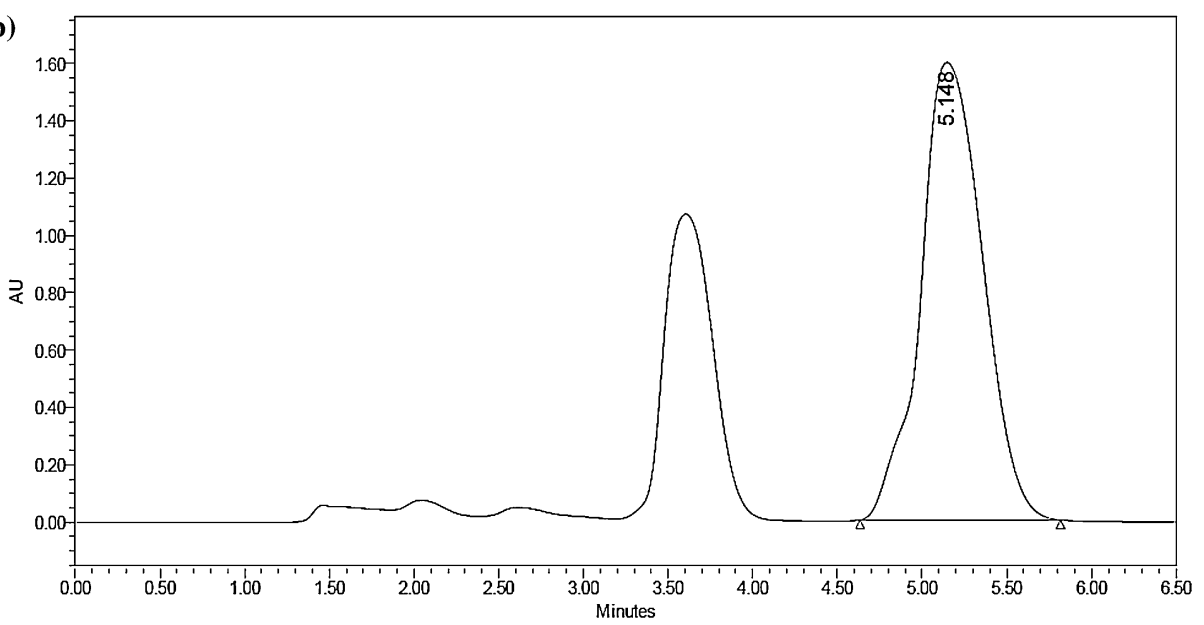


folin-ciocalteu reagent $10 \% \mathrm{v} / \mathrm{v}$ was mixed with $500 \mu \mathrm{l}$ of extract and then $2 \mathrm{~mL}$ the sodium carbonate $7.5 \% \mathrm{w} / \mathrm{v}$ was added. After standing in a dark room for $15 \mathrm{~min}$ was measured the absorbance of the solution at $765 \mathrm{~nm}$ by spectrophotometry (microgram Gallic acid per $100 \mathrm{~g}$ ) [22].

\section{Statistical analysis}

A complete randomized design was used, and the data analyzed by SPSS (ver. 11.0) according to a one-way analysis of variance (ANOVA). The significant differences were obtained by Duncan's multiple range test at the $95 \%$ confidence level. Two replications were carried out for samples with the standard deviation. The curved plotted by the Excel (2013) software.

\section{Results and discussion}

\subsection{Maceration method}

According to optimizing the maceration procedure, which had been applied ethanol concentration of $90 \%$, the temperature of $70^{\circ} \mathrm{C}$ and extraction time of $3.36 \mathrm{~h}$, the amounts of the berberine, phenolic and antioxidant compounds contents from stem were $1.86 \mathrm{mg} \mathrm{g}^{-1}, 11.11 \mathrm{mg}$ Gae $\mathrm{g}^{-1}, 71.84 \%$, respectively [15].

\subsection{QuEChERS method}

QuEChERS has been reported as a high-efficiency extraction method in previous researches. However, it has not been used as a large-scale extraction. It has not been applied in the food industry as well; it is an experimental method.

The amount of berberine reduced to $0.622 \mathrm{mg} \cdot \mathrm{g}^{-1}$ with extraction by standard QuEChERS. But, $1.86 \mathrm{mg} \cdot \mathrm{g}^{-1}$ of the alkaloid extracted by maceration. In addition, phenolic compounds were traces in standard QuEChERS and maceration methods. As a result, the antioxidant activity decreased to $56.37 \%$ from $71.84 \%$ of maceration. Therefore, the QuEChERS was not a suitable method for the extraction of these compounds and maceration had high efficiency compared with the other methods.

\subsection{Modified QuEChERS method}

The results of the modified QuEChERS with ethanol solvent showed that this method contributes to the extraction of phenolic compounds. Ethanolic QuEChERS had higher efficiency in comparison with standard QuEChERS and maceration in the extraction of phenolic compounds.

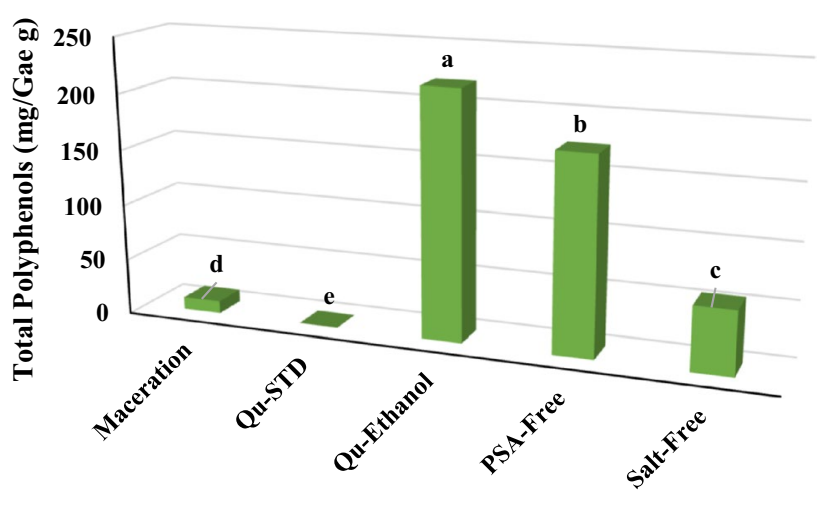

Treatments

Fig.2 The amount of total polyphenols in standard and modified QuEChERS methods

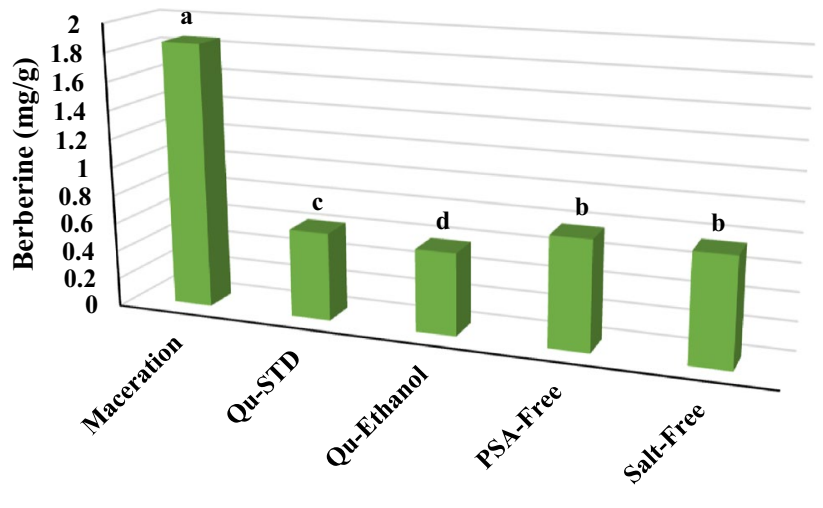

Treatments

Fig.3 The amount of berberine in standard and modified QuEChERS methods

The amount of total phenol of maceration and ethanolic QuEChERS were 11.11 and $215 \mathrm{mg} \mathrm{Gae}^{-1}$, respectively (Fig. 2). This showed that ethanol helped further solubility of phenolic compounds, possibly due to the polarity of the solvent compared to acetonitrile. Therefore, ethanolic QuEChERS can be a suitable method in the food industry, if the purpose is to extract phenolic compounds. However, the method did not significantly influence on the extraction of berberine compounds and antioxidant activity.

For extraction of berberine, no methods had high performance excluding maceration, which was an ideal technique to gain the highest yield of extracting the alkaloid. The salt-free and PSA-free were suitable for the extraction of berberine. Although their efficiency was significantly lower than maceration (Fig. 3).

The investigation of antioxidant activity in different methods showed the salt-free and PSA-free methods had the highest yield. The ethanolic QuECheRS had the 


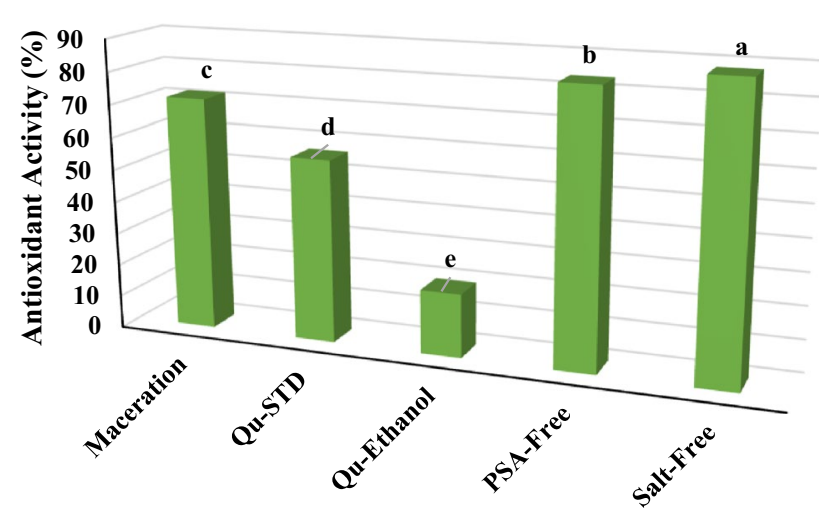

Treatments

Fig.4 The amount of antioxidant activities in standard and modified QuEChERS methods

the high extraction of phenolic compounds (Fig. 4). Although the function of salts and PSA compounds is to remove many of the interfering compounds, it is possible that either the interfering compounds that have antioxidant properties are eliminated or, along with the interfering compounds, the antioxidant compounds are removed.

\section{Conclusion}

The extraction of valuable compounds including berberine, phenolic, and antioxidant compounds of barberry stem was carried out with two extraction methods, namely maceration and QuEChERS, and the yield both of them compared. The results showed that the choice of a targeted approach could effect on extraction efficiency. Besides, using an effective method to extract one compound may not be appropriate for another. Although the QuEChERS method is useful in identify pesticides, this study did not achieve satisfactory results compared with maceration. The amount of berberine extracted with maceration was twice of other methods. However, the ethanolic QuEChERS as a modified method was an efficient method for the extraction of phenolic compounds ( $215 \mathrm{mg} \mathrm{Gae}^{-1}$ ), and can be investigated more for the improvement on a large scale.

Acknowledgements The authors are grateful to Research Institute of Food Science and Technology, Mashhad, Iran, for providing their facilities.

Author contributions All authors read and approved the final manuscript.
Availability of supporting data The datasets used and, or analyzed during the current study are available from the corresponding author on reasonable request.

\section{Compliance with ethical standards}

Conflict of interest The authors declare that they have no conflict of interests.

\section{References}

1. Muhammad M, Jan MR, Shah J, Ara B, Akhtar S, Rahman HR (2017) Evaluation and statistical analysis of the modified QuEChERS method for the extraction of pinoxaden from environmental and agricultural samples. J Anal Sci Technol 8(12):1-10

2. Rejczak T, Tuzimski T (2015) A review of recent developments and trends in the QuEChERS sample preparation approach. Open Chem 13(1):980-1010

3. Lehotay SJ, Son KA, Kwon H, Koesukwiwat U, Fu W, Mastovska K, Hoh E, Leepipatpiboon N (2010) Comparison of QuEChERS sample preparation methods for the analysis of pesticide residues in fruits and vegetables. J Chromatogr A 1217(16):2548-2560

4. ISIRI, Institute of standard and industrial research of Iran Organization, No. 17026(2013) Foods of plant origin-Determination of pesticide residues using GC-MS and/or LC-MS/MS following acetonitrile extraction/partitioning and cleanup by dispersive SPE- QuEChERS-method

5. González-Curbelo M, Socas-Rodríguez B, Herrera-Herrera A, González-Sálamo J, Hernández-Borges J, Rodríguez-Delgado M (2015) Evolution and applications of the QuEChERS method. TrAC, Trends Anal Chem 71:169-185

6. Kafi M Balandari A (2004) Barberry Production and Processing. Ferdowsi University Publication, 1st (Ed.) Mashhad

7. Rahimi-Madiseh M, Lorigoini Z, Zamani-Gharaghoshi H, Rafieian-Kopaei M (2017) Berberis vulgaris specifications and traditional uses. Iran J Basic Med Sci 20(5):569-587

8. Sarraf M, Beig-babaei A, Naji-Tabasi S (2019) Investigating functional properties of barberry species: an overview. J Sci Food Agric 99(12):5255-5269

9. Morton LW, Caccetta RAA, Puddey IB, Croft KD (2000) Chemistry and biological effects of dietary phenolic compounds: relevance to cardiovascular disease. Clin Exp Pharmacol Physiol 27(3):152-159

10. Wu D, Wen W, Qi CL, Zhao RX, Lü JH, Zhong CY, Chen YY (2012) Ameliorative effect of berberine on renal damage in rats with diabetes induced by high-fat diet and streptozotocin. Phytomedicine 19(8-9):712-718

11. Habtemariam S (2011) The therapeutic potential of Berberis darwinii stem-bark: quantification of berberine and in vitro evidence for Alzheimer's disease therapy. Nat prod commun 6(8):1089-1090

12. Amritpal S, Sanjiv D, Navpreet $K$, Jaswinder S (2010) Berberine: alkaloid with wide spectrum of pharmacological activities. J Nat Prod (India) 3(2010):64-75

13. Kosalec I, Gregurek B, Kremer D, Zovko M, Sanković K, Karlović K (2009) Croatian barberry (Berberis croatica Horvat): a new source of berberine analysis and antimicrobial activity. World J Microbiol Biotechnol 25(1):145-150

14. Zarei A, Changizi-Ashtiyani S, Taheri S, Ramezani M (2015) A quick overview on some aspects of endocrinological and therapeutic effects of Berberis vulgaris L. Avicenna J phytomed 5(6):485-497 
15. Sarraf M, Beig-babaei A, Naji-Tabasi S (2020) Optimizing extraction of berberine and antioxidant compounds from barberry by maceration and pulsed electric field-assisted methods. J Berry Res. https://doi.org/10.3233/JBR-200603

16. Valente IM, Santos CM, Moreira MM, Rodrigues JA (2013) New application of the QuEChERS methodology for the determination of volatile phenols in beverages by liquid chromatography. J Chromatogr A 1271(1):27-32

17. Garcia CV, Gotah A (2017) Application of QuEChERS for determining Xenobiotics in foods of animal origin. J Anal Method Chem 2:1-13

18. Tsumoto K, Ejima D, ANNA M, Sencuk M, Kita Y, Arakwa T (2007) Effects of salts on protein-surface interactions: applications for column chromatography. J Pharm Sci 96(7):1677-1699

19. Gupta V, Prakash A, Mathur A (2014) Screening of solvent extracts of Berberis aristata for isolation of anti-inflammatory compound. J Chem Pharm Res 6(6):1196-1206
20. Deepak P, Prativa B, Suri K (2013) Isolation of berberine from Berberis vulgaris Linn. and standardization of aqueous extract by RP-HPLC. International J Herb Med 1(2):106-111

21. Burits $M$, Bucar $F(2000)$ Antioxidant activity of Nigella sativa essential oil. Phytother Res 14(5):323-328

22. McDonald S, Prenzler PD, Antolovich M, Robards K (2001) Phenolic content and antioxidant activity of olive extracts. Food Chem 73(1):73-84

Publisher's Note Springer Nature remains neutral with regard to jurisdictional claims in published maps and institutional affiliations. 